\title{
Influence of aliphatic and aromatic fluorine groups on gas permeability and morphology of fluorinated polyimide films -
}

\section{Supporting Information}

Albert X. Wu ${ }^{1}$, James A. Drayton ${ }^{1}$, Katherine Mizrahi Rodriguez ${ }^{2}$, Qihui Qian ${ }^{1}$, Sharon Lin $^{1}$, and Zachary P. Smith ${ }^{1 *}$

*Corresponding author: zpsmith@mit.edu

${ }^{1}$ Department of Chemical Engineering, Massachusetts Institute of Technology, Cambridge, MA 02139

${ }^{2}$ Department of Materials Science and Engineering, Massachusetts Institute of Technology, Cambridge, MA 02139 
Table S1. GPC results for the four poly(amic acid)s prior to the high temperature cure. The 6FDA-OFB PAA results showed three distinct peaks, representing oligomers of approximately one, two, and three repeat units in size.

\begin{tabular}{|l|l|l|}
\hline & $\mathrm{M}_{\mathrm{n}}(\mathrm{Da})$ & $\mathrm{M}_{\mathrm{w}}(\mathrm{Da})$ \\
\hline 6FDA-6HpDA PAA & 177,000 & 347,000 \\
\hline 6FDA-6FpDA PAA & 110,000 & 214,000 \\
\hline 6FDA-OHB PAA & 114,000 & 186,000 \\
\hline 6FDA-OFB PAA & $600 ; 1,700 ; 3,100$ & $600 ; 1,700 ; 3,100$ \\
\hline 6FDA-OFB film & 56,000 & 138,000 \\
\hline
\end{tabular}


Table S2. Permeation, diffusion, and sorption results for 6FDA-6HpDA.

\begin{tabular}{|c|c|c|c|c|c|c|}
\hline \multicolumn{7}{|c|}{ Permeability (barrer) } \\
\hline $\mathrm{T}\left({ }^{\circ} \mathrm{C}\right)$ & $\mathrm{He}$ & $\mathrm{H}_{2}$ & $\mathrm{O}_{2}$ & $\mathrm{~N}_{2}$ & $\mathrm{CH}_{4}$ & $\mathrm{CO}_{2}$ \\
\hline 35 & $65 \pm 4$ & $65 \pm 4$ & $7.1 \pm 0.4$ & $1.44 \pm 0.09$ & $0.84 \pm 0.05$ & $36 \pm 2$ \\
\hline 45 & $74 \pm 4$ & $73 \pm 4$ & $8.0 \pm 0.5$ & $1.6 \pm 0.1$ & $1.18 \pm 0.07$ & $37 \pm 2$ \\
\hline 55 & $83 \pm 5$ & $81 \pm 5$ & $9.0 \pm 0.5$ & $2.0 \pm 0.1$ & $1.39 \pm 0.08$ & $38 \pm 2$ \\
\hline 65 & $92 \pm 5$ & $90 \pm 5$ & $10.4 \pm 0.6$ & $2.9 \pm 0.2$ & $2.0 \pm 0.1$ & $40 \pm 2$ \\
\hline \multicolumn{7}{|c|}{ Diffusivity $\times 10^{8}\left(\mathrm{~cm}^{2} \mathrm{~s}^{-1}\right)$} \\
\hline $\mathrm{T}\left({ }^{\circ} \mathrm{C}\right)$ & $\mathrm{He}$ & $\mathrm{H}_{2}$ & $\mathrm{O}_{2}$ & $\mathrm{~N}_{2}$ & $\mathrm{CH}_{4}$ & $\mathrm{CO}_{2}$ \\
\hline 35 & - & - & $8.1 \pm 0.6$ & $2.4 \pm 0.2$ & $0.30 \pm 0.02$ & $2.5 \pm 0.2$ \\
\hline 45 & - & - & $12.0 \pm 0.9$ & $3.8 \pm 0.3$ & $0.59 \pm 0.04$ & $3.5 \pm 0.3$ \\
\hline 55 & - & - & $18 \pm 2$ & $7.9 \pm 0.6$ & $1.01 \pm 0.07$ & $5.1 \pm 0.4$ \\
\hline 65 & - & - & $25 \pm 3$ & $20 \pm 2$ & $1.9 \pm 0.1$ & $7.3 \pm 0.5$ \\
\hline \multicolumn{7}{|c|}{ Sorption $\left(\mathrm{cm}^{3}(\mathrm{STP}) \mathrm{cm}^{-3} \mathrm{~atm}^{-1}\right)$} \\
\hline $\mathrm{T}\left({ }^{\circ} \mathrm{C}\right)$ & $\mathrm{He}$ & $\mathrm{H}_{2}$ & $\mathrm{O}_{2}$ & $\mathrm{~N}_{2}$ & $\mathrm{CH}_{4}$ & $\mathrm{CO}_{2}$ \\
\hline 35 & - & - & $0.66 \pm 0.06$ & $0.46 \pm 0.04$ & $2.1 \pm 0.2$ & $11 \pm 1$ \\
\hline 45 & - & - & $0.51 \pm 0.05$ & $0.32 \pm 0.03$ & $1.5 \pm 0.1$ & $8.1 \pm 0.8$ \\
\hline 55 & - & - & $0.39 \pm 0.04$ & $0.20 \pm 0.02$ & $1.0 \quad \pm 0.1$ & $5.7 \pm 0.5$ \\
\hline 65 & - & - & $0.32 \pm 0.04$ & $0.11 \pm 0.01$ & $0.80 \pm 0.07$ & $4.2 \pm 0.4$ \\
\hline
\end{tabular}


Table S3. Permeation, diffusion, and sorption results for 6FDA-6FpDA.

\begin{tabular}{|c|c|c|c|c|c|c|}
\hline \multicolumn{7}{|c|}{ Permeability (barrer) } \\
\hline $\mathrm{T}\left({ }^{\circ} \mathrm{C}\right)$ & $\mathrm{He}$ & $\mathrm{H}_{2}$ & $\mathrm{O}_{2}$ & $\mathrm{~N}_{2}$ & $\mathrm{CH}_{4}$ & $\mathrm{CO}_{2}$ \\
\hline 35 & $114 \pm 6$ & $95 \pm 5$ & $11.8 \pm 0.6$ & $2.5 \pm 0.1$ & $1.29 \pm 0.07$ & $55 \pm 3$ \\
\hline 45 & $127 \pm 7$ & $106 \pm 5$ & $13.0 \pm 0.7$ & $2.9 \pm 0.1$ & $1.60 \pm 0.08$ & $57 \pm 3$ \\
\hline 55 & $141 \pm 7$ & $117 \pm 6$ & $14.4 \pm 0.7$ & $3.5 \pm 0.2$ & $2.0 \pm 0.1$ & $58 \pm 3$ \\
\hline 65 & $156 \pm 8$ & $130 \pm 7$ & $16.3 \pm 0.8$ & $4.5 \pm 0.2$ & $2.9 \pm 0.1$ & $60 \pm 3$ \\
\hline \multicolumn{7}{|c|}{ Diffusivity $\times 10^{8}\left(\mathrm{~cm}^{2} \mathrm{~s}^{-1}\right)$} \\
\hline $\mathrm{T}\left({ }^{\circ} \mathrm{C}\right)$ & $\mathrm{He}$ & $\overline{\mathrm{H}} \mathrm{H}_{2}$ & $\mathrm{O}_{2}$ & $\mathrm{~N}_{2}$ & $\mathrm{CH}_{4}$ & $\mathrm{CO}_{2}$ \\
\hline 35 & - & - & $13 \pm 1$ & $4.1 \pm 0.3$ & $0.59 \pm 0.03$ & $5.3 \pm 0.3$ \\
\hline 45 & - & - & $18 \pm 2$ & $6.4 \pm 0.4$ & $0.96 \pm 0.06$ & $7.0 \pm 0.5$ \\
\hline 55 & - & - & $24 \pm 3$ & $13 \pm 1$ & $2.1 \pm 0.1$ & $8.8 \pm 0.6$ \\
\hline 65 & - & - & $33 \pm 5$ & $23 \pm 3$ & $8.2 \pm 0.6$ & $11.5 \pm 0.9$ \\
\hline \multicolumn{7}{|c|}{ Sorption $\left(\mathrm{cm}^{3}(\mathrm{STP}) \mathrm{cm}^{-3} \mathrm{~atm}^{-1}\right)$} \\
\hline $\mathrm{T}\left({ }^{\circ} \mathrm{C}\right)$ & $\mathrm{He}$ & $\mathrm{H}_{2}$ & $\mathrm{O}_{2}$ & $\mathrm{~N}_{2}$ & $\mathrm{CH}_{4}$ & $\mathrm{CO}_{2}$ \\
\hline 35 & - & - & $0.68 \pm 0.07$ & $0.47 \pm 0.04$ & $1.7 \pm 0.1$ & $7.9 \pm 0.6$ \\
\hline 45 & - & - & $0.56 \pm 0.06$ & $0.34 \pm 0.03$ & $1.3 \pm 0.1$ & $6.2 \pm 0.5$ \\
\hline 55 & - & - & $0.46 \pm 0.06$ & $0.21 \pm 0.02$ & $0.72 \pm 0.06$ & $5.0 \pm 0.4$ \\
\hline 65 & - & - & $0.38 \pm 0.06$ & $0.15 \pm 0.02$ & $0.26 \pm 0.02$ & $4.0 \pm 0.4$ \\
\hline
\end{tabular}


Table S4. Permeation, diffusion, and sorption results for 6FDA-OHB.

\begin{tabular}{|c|c|c|c|c|c|c|}
\hline \multicolumn{7}{|c|}{ Permeability (barrer) } \\
\hline $\mathrm{T}\left({ }^{\circ} \mathrm{C}\right)$ & $\mathrm{He}$ & $\mathrm{H}_{2}$ & $\mathrm{O}_{2}$ & $\mathrm{~N}_{2}$ & $\mathrm{CH}_{4}$ & $\mathrm{CO}_{2}$ \\
\hline 35 & $59 \pm 3$ & $58 \pm 3$ & $6.4 \pm 0.4$ & $1.23 \pm 0.07$ & $0.81 \pm 0.05$ & $35 \pm 2$ \\
\hline 45 & $67 \pm 4$ & $65 \pm 4$ & $7.2 \pm 0.4$ & $1.51 \pm 0.08$ & $1.13 \pm 0.06$ & $36 \pm 2$ \\
\hline 55 & $75 \pm 4$ & $72 \pm 4$ & $8.3 \pm 0.5$ & $1.9 \pm 0.1$ & $1.49 \pm 0.08$ & $38 \pm 2$ \\
\hline 65 & $84 \pm 5$ & $81 \pm 5$ & $9.8 \pm 0.5$ & $2.8 \pm 0.2$ & $2.0 \pm 0.1$ & $41 \pm 2$ \\
\hline \multicolumn{7}{|c|}{ Diffusivity $\times 10^{8}\left(\mathrm{~cm}^{2} \mathrm{~s}^{-1}\right)$} \\
\hline $\mathrm{T}\left({ }^{\circ} \mathrm{C}\right)$ & $\mathrm{He}$ & $\overline{\mathrm{H}} \mathrm{H}_{2}$ & $\mathrm{O}_{2}$ & $\mathrm{~N}_{2}$ & $\mathrm{CH}_{4}$ & $\mathrm{CO}_{2}$ \\
\hline 35 & - & - & $7.4 \pm 0.5$ & $1.8 \pm 0.1$ & $0.24 \pm 0.02$ & $2.3 \pm 0.1$ \\
\hline 45 & - & - & $9.5 \pm 0.7$ & $3.3 \pm 0.2$ & $0.38 \pm 0.02$ & $3.2 \pm 0.2$ \\
\hline 55 & - & - & $12 \pm 1$ & $6.8 \pm 0.5$ & $0.68 \pm 0.04$ & $4.4 \pm 0.3$ \\
\hline 65 & - & - & $23 \pm 3$ & $16 \pm 1$ & $1.21 \pm 0.08$ & $6.6 \pm 0.5$ \\
\hline \multicolumn{7}{|c|}{ Sorption $\left(\mathrm{cm}^{3}(\mathrm{STP}) \mathrm{cm}^{-3} \mathrm{~atm}^{-1}\right)$} \\
\hline $\mathrm{T}\left({ }^{\circ} \mathrm{C}\right)$ & $\mathrm{He}$ & $\mathrm{H}_{2}$ & $\mathrm{O}_{2}$ & $\mathrm{~N}_{2}$ & $\mathrm{CH}_{4}$ & $\mathrm{CO}_{2}$ \\
\hline 35 & - & - & $0.66 \pm 0.06$ & $0.52 \pm 0.04$ & $2.6 \pm 0.2$ & $11 \pm 1$ \\
\hline 45 & - & - & $0.58 \pm 0.05$ & $0.35 \pm 0.03$ & $2.2 \pm 0.2$ & $8.6 \pm 0.7$ \\
\hline 55 & - & - & $0.48 \pm 0.05$ & $0.22 \pm 0.02$ & $1.7 \pm 0.1$ & $6.6 \pm 0.6$ \\
\hline 65 & - & - & $0.32 \pm 0.04$ & $0.13 \pm 0.01$ & $1.2 \pm 0.1$ & $4.7 \pm 0.4$ \\
\hline
\end{tabular}


Table S5. Permeation, diffusion, and sorption results for 6FDA-OFB.

\begin{tabular}{|c|c|c|c|c|c|c|}
\hline \multicolumn{7}{|c|}{ Permeability (barrer) } \\
\hline $\mathrm{T}\left({ }^{\circ} \mathrm{C}\right)$ & $\mathrm{He}$ & $\mathrm{H}_{2}$ & $\mathrm{O}_{2}$ & $\mathrm{~N}_{2}$ & $\mathrm{CH}_{4}$ & $\mathrm{CO}_{2}$ \\
\hline 35 & $220 \pm 20$ & $220 \pm 20$ & $36 \pm 3$ & $9.7 \pm 0.7$ & $5.2 \pm 0.4$ & $160 \pm 10$ \\
\hline 45 & $230 \pm 20$ & $230 \pm 20$ & $38 \pm 3$ & $10.1 \pm 0.7$ & $5.5 \pm 0.4$ & $150 \pm 10$ \\
\hline 55 & $250 \pm 20$ & $240 \pm 20$ & $39 \pm 3$ & $11.1 \pm 0.8$ & $6.2 \pm 0.4$ & $150 \pm 10$ \\
\hline 65 & $270 \pm 20$ & $250 \pm 20$ & $40 \pm 3$ & $12.7 \pm 0.9$ & $7.5 \pm 0.5$ & $140 \pm 10$ \\
\hline \multicolumn{7}{|c|}{ Diffusivity $\times 10^{8}\left(\mathrm{~cm}^{2} \mathrm{~s}^{-1}\right)$} \\
\hline $\mathrm{T}\left({ }^{\circ} \mathrm{C}\right)$ & $\mathrm{He}$ & $\overline{\mathrm{H}} \mathrm{H}_{2}$ & $\mathrm{O}_{2}$ & $\mathrm{~N}_{2}$ & $\overline{\mathrm{CH}} 4$ & $\overline{\mathrm{CO}}$ \\
\hline 35 & - & - & $25 \pm 3$ & $7.9 \pm 0.7$ & $1.6 \pm 0.1$ & $8.9 \pm 0.8$ \\
\hline 45 & - & - & $33 \pm 4$ & $11 \pm 1$ & $2.0 \pm 0.2$ & $11 \pm 1$ \\
\hline 55 & - & - & $42 \pm 6$ & $19 \pm 2$ & $3.6 \pm 0.3$ & $14 \pm 1$ \\
\hline 65 & - & - & $60 \pm 10$ & $33 \pm 4$ & $9.6 \pm 0.9$ & $18 \pm 2$ \\
\hline \multicolumn{7}{|c|}{ Sorption $\left(\mathrm{cm}^{3}(\mathrm{STP}) \mathrm{cm}^{-3} \mathrm{~atm}^{-1}\right)$} \\
\hline $\mathrm{T}\left({ }^{\circ} \mathrm{C}\right)$ & $\mathrm{He}$ & $\mathrm{H}_{2}$ & $\mathrm{O}_{2}$ & $\mathrm{~N}_{2}$ & $\mathrm{CH}_{4}$ & $\mathrm{CO}_{2}$ \\
\hline 35 & - & - & $1.1 \pm 0.1$ & $0.9 \pm 0.1$ & $2.5 \pm 0.3$ & $14 \quad \pm 2$ \\
\hline 45 & - & - & $0.9 \pm 0.1$ & $0.67 \pm 0.08$ & $2.1 \pm 0.2$ & $10 \pm 1$ \\
\hline 55 & - & - & $0.7 \pm 0.1$ & $0.45 \pm 0.06$ & $1.3 \pm 0.1$ & $8 \pm 1$ \\
\hline 65 & - & - & $0.5 \pm 0.1$ & $0.30 \pm 0.04$ & $0.59 \pm 0.07$ & $6.1 \pm 0.7$ \\
\hline
\end{tabular}




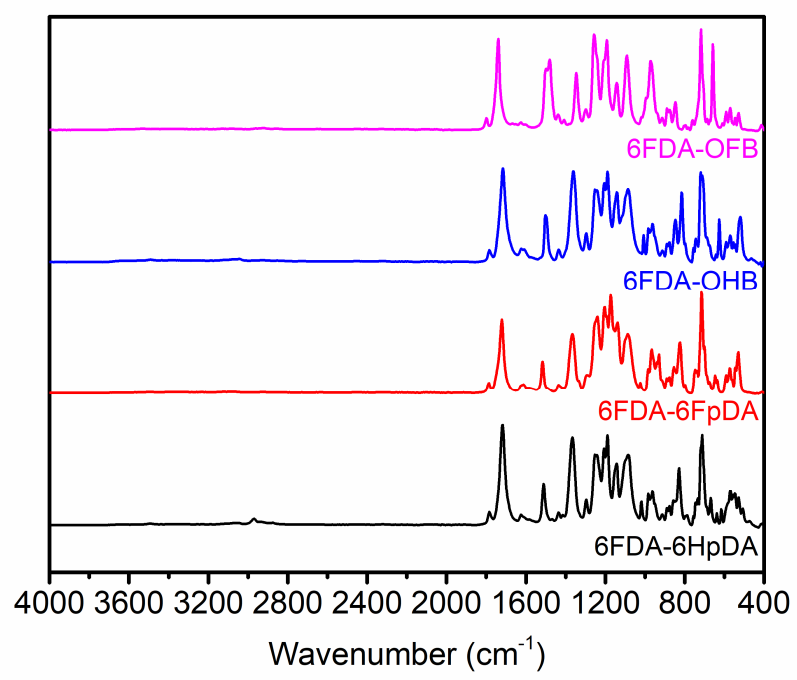

Figure S1. FTIR spectra for the four polyimide films. 

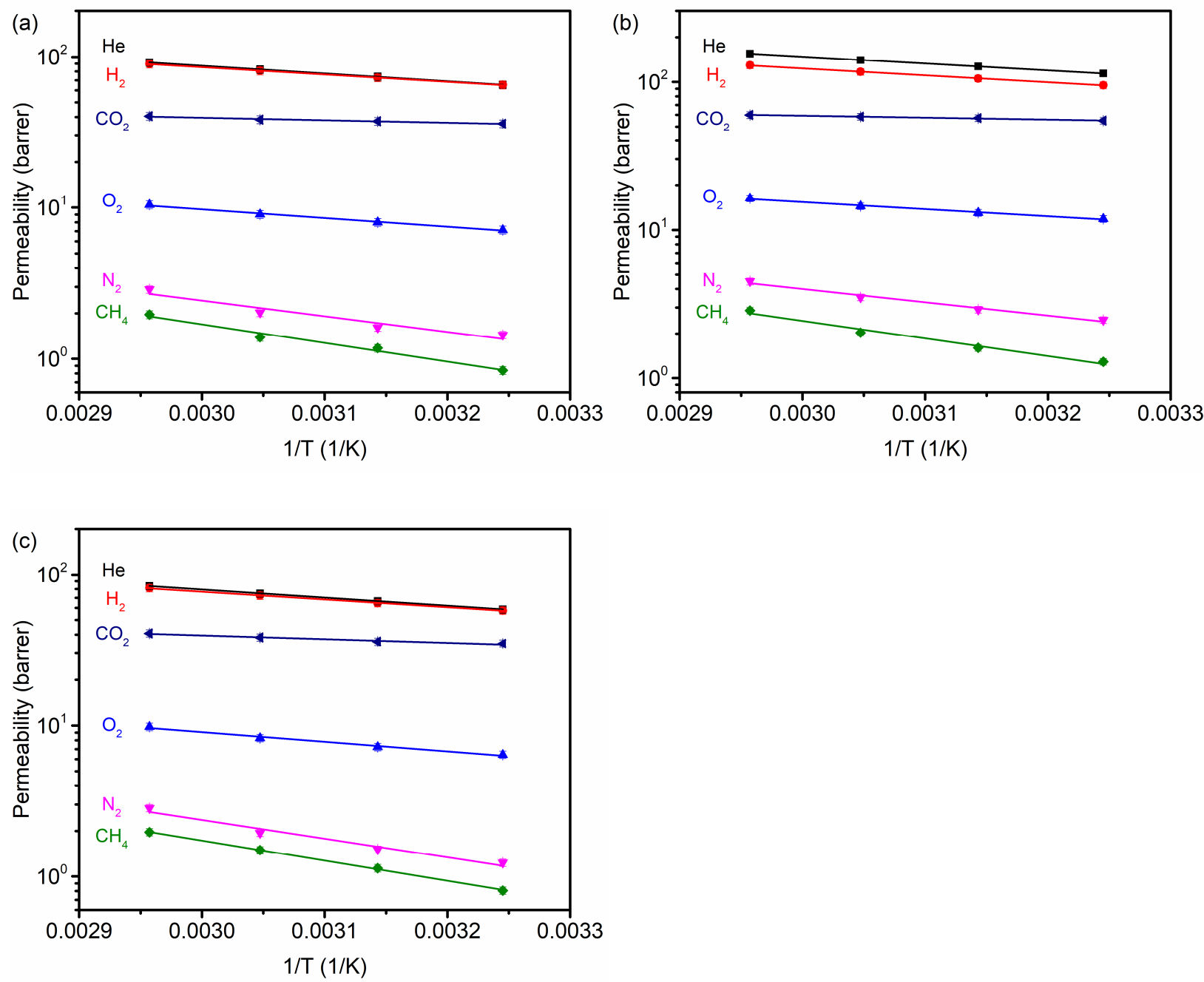

Figure S2. Arrhenius plots showing the effect of temperature on permeability for (a) 6FDA6HpDA, (b) 6FDA-6FpDA, and (c) 6FDA-OHB measured at 35, 45, 55, and $65^{\circ} \mathrm{C}$ and 15 psi. 


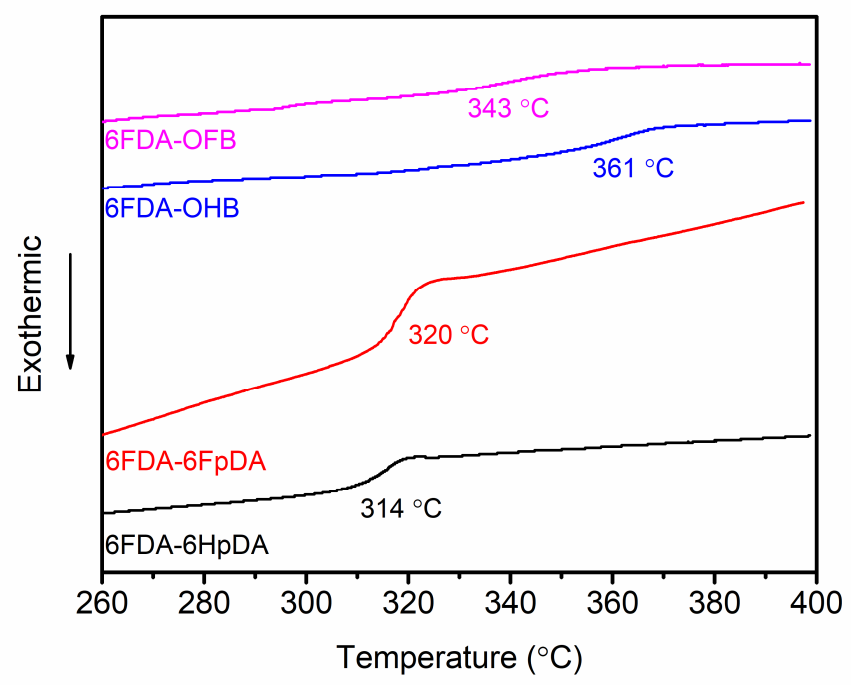

Figure S3. DSC results for the four polyimide films. The results displayed are the last scan for each polymer.

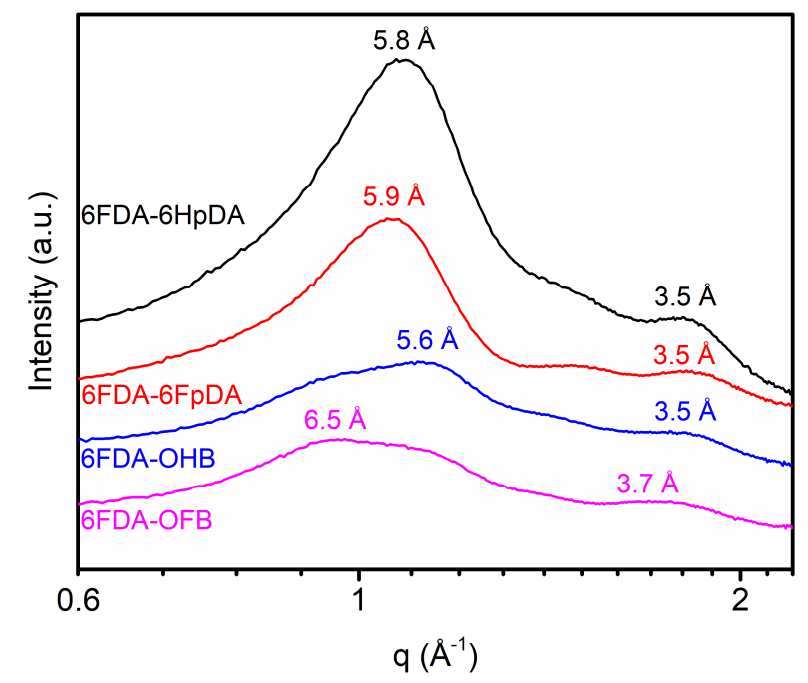

Figure S4. WAXS results for the four polyimide films. 